\section{Alimentação do recém-nascido pré-termo: métodos alternativos de transição da gavagem para o peito materno}

\section{The feeding of preterm newborns: alternative methods for the transition from tube-feeding to breastfeeding}

Rebeca Raposo Aquino 1 Mônica Maria Osório 2

\begin{abstract}
This study investigates the methods normally used to effect the transition from tube-feeding to full breastfeeding in preterm infants. The information was gathered from papers catalogued in the Lilacs, Medline, and SciElo databases, between 1994 and 2007. Four articles were selected and only randomized studies considered (three of them, being controlled). The literature describes a number of methods used to effect the transition from tubefeeding to full breastfeeding: bottle-feeding and cupfeeding. The studies demonstrate that the babies who used cup-feeding obtained better results in relation to physiological stability (cardiac frequency and oxygen saturation) and to the impact on full breastfeeding on being discharged from hospital. Given the scarcity of studies in the literature and the methodological problems found, it is clear that more studies need to be carried out to compare the alternative methods used for the preterm feeding plan.
\end{abstract}

Key words Premature, Feeding methods, Bottle feeding, Cup feeding
1 Programa de Pós-Graduação em Saúde da Criança e do Adolescente. Universidade Federal de Pernambuco. Recife, PE, Brasil.

2 Departamento de Nutrição. Universidade Federal de Pernambuco. Rua Moraes Rego, s.n. Cidade Universitária. Recife, PE, Brasil. CEP: 50.670-901.E-mail: mosorio@ufpe.br

\section{Resumo}

O objetivo deste trabalho foi revisar os métodos de alimentação mais utilizados na transição da gavagem para o peito materno em recém-nascidos pré-termo. A pesquisa foi realizada nas bases de dados: Lilacs, Medline, SciElo, no período de 1994 a 2007. Foram selecionados quatro artigos, considerando apenas os estudos randomizados (três dos quais também controlados). A literatura pesquisada descreve alguns desses métodos de transição da alimentação: mamadeira e copinho. Os estudos demonstram que os bebês que utilizaram copinho apresentaram melhores resultados em relação à estabilidade fisiológica (freqüencia cardíaca e saturação de oxigenio) e ao impacto no aleitamento materno exclusivo na alta hospitalar. Devido à escassez de estudos na literatura e aos problemas metodológicos encontrados, faz-se necessária a realização de mais estudos para comparar a utilização dos métodos de transição da alimentação em recém-nascidos prétermo.

Palavras-chave Prematuro, Métodos de alimentação, Mamadeira, Copinho 


\section{Introdução}

A freqüência e a duração do aleitamento materno são mais baixas nos recém-nascidos pré-termo (RNPT) do que nos a termo. Isso acontece, principalmente, por dois fatores: a dificuldade no estabelecimento e na manutenção de uma produção eficiente de leite por parte da mãe do pré-termo, ocasionada, entre outros motivos, pelo estresse a que é exposta e pelo afastamento do seu bebê devido a presença de doenças neonatais freqüentemente associadas à prematuridade; e a maneira como é realizada a transição da alimentação. ${ }^{1}$

No início da sua vida, na unidade neonatal, o recém-nascido pré-termo pode necessitar de um maior suporte alimentar dependendo da sua gravidade, tendo que receber os primeiros nutrientes através da alimentação parenteral (intravenosa). Após a sua melhora nutricional, o leite materno ordenhado ou uma fórmula láctea específica é oferecida através de uma sonda orogástrica ou nasogástrica e o volume da alimentação é aumentado de forma lenta e gradual, proporcionando um tempo necessário para a adaptação do sistema gastrintestinal, ainda imaturo. ${ }^{2}$

Além da imaturidade do sistema gastrintestinal, a sincronia entre sucção, deglutição e respiração é essencial para o sucesso da alimentação oral. ${ }^{3} \mathrm{~A}$ sucção e a deglutição, também imaturas, requerem a integração das atividades musculares dos lábios, bochechas, mandíbula, língua, palato, faringe e laringe. ${ }^{4}$ Enquanto não houver essa integração, o recém-nascido pré-termo inicialmente se alimenta por gavagem, para posteriormente se estabelecer o aleitamento no peito. Não sendo o bebê capaz de mamar no peito, faz-se necessária a utilização de métodos alternativos para alimentação. ${ }^{5}$

A forma como o leite é oferecido para os recémnascidos pré-termo é uma variável importante a ser considerada. A transição da alimentação constitui uma grande dificuldade para o binômio mãe-filho, por ser uma mudança importante para um bebê frágil e que ainda não estava preparado para nascer. 2,6 Existem vários métodos para realizar essa transição. 5 O bebê precisa aprender a se alimentar, de forma segura e prazerosa, o mais rápido possível e é importante que o método de transição seja adequado a fim de garantir o sucesso da amamentação.

O presente estudo tem como objetivo revisar os métodos de alimentação mais utilizados na transição da gavagem para o peito materno em recém-nascidos pré-termo, descrevendo as vantagens e desvantagens desses métodos e a influência nos resultados do aleitamento materno. Considerando as dificuldades para o início da amamentação em recém-nascidos pré-termo, espera-se que o presente trabalho venha sensibilizar e contribuir para reflexão dos profissionais de saúde que prestam assistência a esses bebês em unidades neonatais.

\section{Métodos}

A pesquisa foi realizada nas bases de dados eletrônicos, objetivando identificar estudos nacionais e internacionais sobre os métodos alternativos de alimentação utilizados na transição da gavagem para o peito materno em recém-nascidos pré-termo. As bases de dados consultadas foram: Literatura Latino-Americana e do Caribe em Ciências da Saúde (Lilacs), Medline e Scientific Eletronic Library On-line (SciElo), no período de 1994 a 2007. Foram selecionados os artigos nos idiomas inglês, português e espanhol. Os indexadores utilizados em inglês foram: preterm, feeding methods, bottle feeding e cup feeding.

Inicialmente foram selecionados nove artigos, sendo oito estrangeiros5-12 e um brasileiro. 13 Em seguida, foi realizada uma nova seleção considerando apenas os estudos randomizados, restando apenas quatro artigos.10-13 Os artigos foram revisados segundo o ano de publicação, desenho do estudo, amostra, métodos, objetivos e resultados como mostrado na Tabela 1 .

\section{Resultados}

Muitos investigadores têm estudado os efeitos das diferentes formas de alimentar o bebê. Marinelli et al., ${ }^{10}$ nos Estados Unidos, com o objetivo de comparar o copinho em relação à mamadeira, realizaram um estudo prospectivo e randomizado em dois grupos (copinho e mamadeira), com 56 recémnascidos pré-termo, os quais as mães tinham intenção de amamentar. As freqüências cardíacas e respiratórias e a saturação de oxigênio foram verificadas antes e durante as duas primeiras alimentações por via oral, assim como o volume ingerido, o tempo gasto para completar a alimentação e as intercorrências (apnéia, bradicardia, engasgos ou vômito). Os resultados demonstraram que durante o uso do copinho os bebês permaneciam mais estáveis do que os que usaram a mamadeira, em relação à freqüência cardíaca $(p<0,001)$, saturação de oxigênio $(p=0,002)$, e que não houve diferenças entre os métodos em relação à freqüência respiratória e intercorrências. Entretanto, os bebês do copinho ingeriram menos 
Estudos sobre os métodos de alimentação do recém-nascido pré-termo, no período de 1994 a 2004, de acordo com autor, desenho do estudo, amostra, e métodos e os objetivos.

\begin{tabular}{|c|c|c|c|c|}
\hline Autor & $\begin{array}{l}\text { Desenho do } \\
\text { estudo }\end{array}$ & Amostra & Métodos & Objetivos \\
\hline $\begin{array}{l}\text { Marinelli et al.,10 } \\
\text { (Estados Unidos em } \\
\text { 2001). }\end{array}$ & $\begin{array}{l}\text { Estudo } \\
\text { prospectivo e } \\
\text { randomizado }\end{array}$ & $\begin{array}{l}56 \text { recém-nascidos } \\
\text { pré-termo de mães } \\
\text { com intenção de } \\
\text { amamentar. }\end{array}$ & $\begin{array}{l}\text { Copinho e } \\
\text { mamadeira }\end{array}$ & $\begin{array}{l}\text { Verificar as freqüências } \\
\text { cardíacas e respiratórias, a } \\
\text { saturação de oxigênio, o } \\
\text { volume ingerido, o tempo } \\
\text { gasto para completar a } \\
\text { alimentação e as intercor- } \\
\text { rências (apneia, bradicar- } \\
\text { dia, engasgos ou vômitos) } \\
\text { antes e durante a alimen- } \\
\text { tação com o copinho e } \\
\text { com a mamadeira. }\end{array}$ \\
\hline $\begin{array}{l}\text { Mosley et al.,11 } \\
\text { (Reino Unido, 2001). }\end{array}$ & $\begin{array}{l}\text { Estudo clínico } \\
\text { randomizado } \\
\text { prospectivo }\end{array}$ & $\begin{array}{l}14 \text { recém-nascidos } \\
\text { pré-termo }\end{array}$ & $\begin{array}{l}\text { Mamadeira } \\
\text { e copinho }\end{array}$ & $\begin{array}{l}\text { Comparar o impacto do } \\
\text { copinho e da mamadeira } \\
\text { no aleitamento materno }\end{array}$ \\
\hline
\end{tabular}

Collins et al., 12

(Austrália, 2004).

Rocha et al.,13

(Ceará, Brasil, 2002).
Estudo

randomizado controlado
303 recém-nascidos pré-termo6, Idade gestacional 23-33 semanas.
Estudo

experimental controlado, randomizado
78 recém-nascidos pré-termo Idade gestacional 32-

36 semanas e peso ao nascimento $<1700$
Mamadeira, copinho e chupeta
Determinar o efeito de bicos artificiais (mamadeira e chupeta) e do copinho no aleitamento materno em recém-nascidos pré-termo, verificando o tipo de alimentação no momento da alta, a prevalência de aleitamento materno no peito três e seis meses após a alta e o tempo de permanência no hospital.

Copinho e mamadeira

Comparar o impacto da mamadeira e do copinho no ganho de peso, na saturação de oxigênio e no aleitamento materno.
Resultados

O grupo do copinho apresentou melhores resultados em relação à freqüência cardíaca e saturação de oxigênio. Não houve diferença em relação à freqüência respiratória e intercorrências entre os dois grupos. O grupo do copinho ingeriu menos volume e demorou mais tempo para completar a alimentação.

Não foi observada diferença estatisticamente significante nos dois grupos em relação ao aleitamento materno.

Não houve influência da chupeta em relação ao aleitamento materno. $\mathrm{O}$ uso do copinho aumenta as chances do aleitamento materno exclusivo no momento da alta hospitalar. 0 copinho esteve associado à longa permanência hospitalar.

Não houve diferenças em relação ao tempo de alimentação, ganho de peso e aleitamento materno na alta hospitalar nos dois grupos. Houve diferença significante em relação à episódios de queda na saturação de oxigênio e no aleitamento materno aos três meses. 
volume $(p=0,001)$ e demoraram mais tempo $(p=0,002)$ para completar a alimentação. Ao final do estudo, os pesquisadores concluíram que o copinho pode ser considerado um método de alimentação seguro para bebês pré-termo enquanto esses aprendem a mamar. Entretanto, não foi objetivo do estudo verificar qual o melhor método de transição em relação ao aleitamento materno. Tais autores referem, como uma das limitações do estudo, a impossibilidade de quantificar o número de vezes que cada criança estudada foi levada ao peito materno antes das duas primeiras alimentações por via oral, e se isso pode ter influenciado o desempenho dela na alimentação.

Mosley et al.11 realizaram um estudo clínico randomizado, durante três meses, com todos os recém-nascidos pré-termo da unidade de cuidados especiais de um hospital no Reino Unido que preenchessem os critérios de inclusão do estudo: bebês nascidos de mães que expressavam o desejo de amamentar e não tinham preferência pelo copinho ou mamadeira como método de alimentação complementar, idade gestacional ao nascimento entre $30 \mathrm{e}$ 37 semanas, ausência de má-formação congênita, não tivessem tido contato com o copinho ou mamadeira antes da admissão no estudo. Foram acompanhados 14 recém-nascidos pré-termo com o objetivo de comparar o impacto do copinho e da mamadeira no aleitamento materno. Os bebês foram alocados de forma randomizada em dois grupos: o que recebeu complemento pelo copinho e o que recebeu pela mamadeira. No momento da alta foram avaliadas as formas de alimentação e não foi observada diferença estatisticamente significante nos dois grupos em relação ao aleitamento materno. Entretanto, o estudo se tornou inconclusivo pelo tamanho pequeno da amostra, sendo recomendável a sua repetição em uma amostra de maior tamanho.

Rocha et al.,13 em um estudo experimental controlado e randomizado realizado em São Paulo, entre 1998 e 2000, investigaram o impacto da alimentação com mamadeira e com copinho em 78 recém-nascidos pré-termo, segundo o tempo de alimentação, problemas durante a alimentação, ganho de peso, a saturação de oxigênio e as taxas de aleitamento materno. Os resultados não encontraram diferenças estatisticamente significantes nestes grupos em relação ao tempo de alimentação $(13,4 v \mathrm{~s}$ $11,8 \mathrm{~min})$, ganho de peso $(14,7$ vs $14,1 \mathrm{~g} / \mathrm{Kg} / \mathrm{dia})$ e à prevalência do aleitamento materno na alta $(79,4 \%$ vs $81,8 \%)$ e durante os três primeiros meses $(44,1 \%$ vs $43,2 \%)$. Entretanto, foram observados efeitos benéficos do copinho quando comparado à mamadeira, tais como: menor incidência de bebês com episódios de queda na saturação de oxigênio durante a alimentação (13,6\% para o copinho e $35,3 \%$ para a mamadeira, $p=0,02)$ e maior prevalência do aleitamento materno aos três meses de idade naqueles que ainda estavam em aleitamento materno na primeira visita de retorno, entre o quinto e o décimo quinto dia após a alta $(68,4 \%$ para o copinho e $33,3 \%$ para a mamadeira, $p=0,04)$. Esses autores também observaram que o uso do copinho não ocasiona intercorrências (broncoaspiração e apnéia).

Collins et al. 12 realizaram um estudo controlado e randomizado, com objetivo de determinar o efeito de bicos artificiais (mamadeira e chupeta) e do copinho no aleitamento materno em recém-nascidos pré-termo, verificando o tipo de alimentação no momento da alta (aleitamento exclusivo, aleitamento parcial ou sem aleitamento), a prevalência de aleitamento materno no peito aos três e seis meses após a alta e o tempo de permanência no hospital. Foram recrutados 303 pré-termos, de dois hospitais terciários na Austrália, com idade gestacional ao nascimento entre 23-33 semanas. Esses bebês foram randomizados em um dos quatro grupos seguintes: copinho/chupeta $(n=69)$, copinho/sem chupeta $(\mathrm{n}=82)$, mamadeira/chupeta $(\mathrm{n}=82)$ e mamadeira/sem chupeta $(n=70)$. O copinho e a mamadeira foram utilizados quando a mãe não estava presente para amamentar o seu bebê ou quando era necessário oferecer um complemento por via oral. Os bebês dos grupos da chupeta iniciaram o seu uso quando entravam na pesquisa, durante a alimentação por gavagem ou enquanto descansavam. Os resultados demonstraram não haver uma diferença estatisticamente significante em relação ao aleitamento materno entre os grupos da chupeta e sem chupeta, no momento da alta $(p=0,5)$, aos três meses $(p=0,98)$ e aos seis meses $(p=0,51)$ após a alta. Quando os grupos do copinho e da mamadeira foram comparados, houve uma diferença estatística significante no momento da alta hospitalar $(p=0,03)$, sendo o copinho mais positivo em relação ao aleitamento materno. Entretanto, não houve diferenças significantes após a alta hospitalar. O uso da chupeta não influenciou o tempo de permanência no hospital, mas o do copinho sim. Os bebês do grupo do copinho permaneceram mais tempo no hospital $(p=0,01)$. Os autores concluíram que a chupeta não interfere no aleitamento materno em recém-nascidos pré-termo, e que o uso do copinho aumenta, de forma significativa, as chances de o bebê estar mamando exclusivamente no momento da alta hospitalar. A limitação desse estudo foi a pobre adesão na intervenção, ou seja, nem todos os indivíduos da amostra 
cumpriram as instruções corretamente, por sentimentos negativos, em particular, relacionados ao uso do copinho e da chupeta, o que reduz o poder de identificar o real efeito desses na amamentação.

\section{Discussão}

Os estudos encontrados na literatura fazem comparação entre os métodos alternativos de alimentação em recém-nascidos pré-termo, sendo referenciados o copinho e a mamadeira. De maneira geral, discutem as vantagens e desvantagens desses métodos em relação à estabilidade fisiológica e ao impacto no aleitamento materno exclusivo. Em dois dos estudos analisados, foi encontrada uma melhor estabilidade clínica naqueles bebês que utilizaram copinho quando comparados aos de mamadeira, sendo relatadas diferenças significantes em termos de menor incidência na queda de saturação de oxigênio e no aumento da freqüência cardíaca. 10,13

Dos três estudos11-13 que compararam o uso do copinho e da mamadeira no aleitamento materno exclusivo, na alta hospitalar, apenas um 12 mostrou impacto na incidência do aleitamento materno, apresentando diferença estatisticamente significante. Ressalta-se que em um dos trabalhos, 11 o pequeno tamanho amostral pode ter comprometido a conclusão.

Neifert et al.14 relataram que experiências orais precoces com bicos artificiais favorecem o surgimento de mecanismos de sucção diferentes dos utilizados no aleitamento materno, podendo causar alteração na pega e, conseqüentemente, fracasso no aleitamento pela "confusão de bicos". Essa é definida como a dificuldade do bebê em atingir a configuração oral correta (abertura de boca e posicionamento da língua), a pega adequada e o padrão de sucção, necessários para um aleitamento materno eficiente, depois de exposição à mamadeira ou a qualquer outro bico artificial.

Acredita-se que a alimentação com o copinho seja fácil para o bebê pré-termo pela hipótese de que ele é capaz de regular a ingestão do leite, não precisando do "trabalho" de sugar. 6 Enquanto o leite é oferecido pelo copinho, o bebê controla o ritmo sucção/lambida, o que proporciona uma respiração adequada, fazendo com que a deglutição ocorra no momento certo. Como resultado, há um menor gasto de energia. 5 Entretanto, o estudo de Marinelli et al. 10 demonstrou que o uso do copinho leva o bebê a ingerir menos volume, por mais derramamento do leite, e demorar mais tempo para completar a alimentação.

Em conclusão, é possível verificar que a literatura sobre o tema é escassa. Diante disso, existe a necessidade da realização de estudos, com desenhos metodológicos adequados, para se comparar a utilização dos métodos de transição da alimentação em recém-nascidos pré-termo. Salienta-se também que, atualmente, há outros métodos de transição, tais como translactação, 15 paladai, 16 , finger feeding, 17 que precisam de fundamentações científicas, para serem utilizados de maneira mais segura pelos profissionais de saúde que atuam diretamente com os cuidados a esses recém-nascidos.

\section{Referências}

1. Callen J, Pinelli J. A review of the literature examining the benefits and challenges, incidence and duration, and barriers to breastfeeding in preterm infants. Adv Neonatal Care. 2005; 5: 72-88.

2. Brock R. Recém-nascido prematuro, baixo peso e retardo do crescimento intra-uterino. In: Bassetto MC. Neonatologia, um convite à atuação fonoaudiológica. São Paulo: Lovise; 1998. p. 67-73.

3. Morris SE, Klein MD. The child who is premature. In: Morris SE, Klein MD. Pre-feeding skills: a comprehensive resource for mealtime development. San Antonio, Texas: Therapy Skill Builders; 2000. p. 537-52.

4. Gewolb IH, Vice FL, Schweitzer-Kenney EL, Taciak VL, Bosma JF. Developmental patterns of rhythmic suck and swallow in preterm infants. Dev Med Child Neurol. 2001; 43: $22-7$.

5. Gupta A, Khanna K, Chattree S. Cup feeding: an alternative to bottle feeding in a neonatal intensive care unit. J Trop Pediatr. 1999; 45: 108-10.
6. Dowling DA, Meier PP, Difiore JM, Blatz MA, Martin RJ. Cup-feeding for preterm infants: mechanics and safety. $J$ Hum Lact. 2002; 18: 13-20.

7. Lang S, Lawrence CJ, L'E Orme R. Cup feeding: an alternative method of infant feeding. Arch Dis Child. 1994; 71: 365-9.

8. Malhotra N, Vishwambaran L, Sundaram KR, Narayanan I. A controlled trial of alternative methods of oral feeding in neonates. Early Hum Dev. 1999; 54: 29-38.

9. Chen C, Wang T, Chang H, Chi C. The effect of breast- and bottle-feeding on oxygen saturation and body temperature in preterm infants. J Hum Lact. 2000; 16: 21-7.

10. Marinelli KA, Burke GS, Dodd VL. A comparison of the safety of cupfeedings and bottlefeedings in premature infants whose mothers intend to breastfeed. J Perinatol. 2001; 21: 350-5. 
11. Mosley C, Whittle C, Hicks C. A pilot study to assess the viability of a randomized controlled trial of methods of supplementary feeding of breastfed pre-term babies. Midwifery. 2001; 17: 150-7.

12. Collins CT, Ryan P, Crowther CA, McPhee AJ, Paterson S, Hiller JE. Effect of bottles, cups, and dummies on breast feeding in preterm infants: a randomised controlled trial. BMJ. 2004; 329: 93-8.

13. Rocha NMN, Martinez FE, Jorge SM. Cup or bottle for preterm infants: effects on oxygen saturation, weight gain, and breastfeeding. J Hum Lact. 2002; 18: 132-8.

14. Neifert M, Lawrence R, Seacat J. Nipple confusion: toward a formal definition. J Pediatr. 1995; 126: 125-9.

Recebido em 10 de julho de 2006

Versão final apresentada em 6 de outubro de 2007

Aprovado em 17 de novembro de 2007
15. Lima GSM, Aquino RR. Avaliação da técnica da translactação em 362 recém-nascidos pré-termo admitidos na unidade canguru-IMIP. In: Anais do XVII Congresso Brasileiro de Perinatologia; 2001 10-14 de nov.; Florianópolis, Santa Catarina: Sociedade Brasileira de Pediatria; 2001. p. 163.

16. Malhotra N, Vishwambaran L, Sundaram KR, Narayanan I. A controlled trial of alternative methods of oral feeding in neonates. Early Hum Dev. 1999; 54: 29-38.

17. Oddy WH, Glenn K. Implementing the Baby Friendly Hospital Initiative: the role of finger feeding. Breastfeed Rev. 2003; 11: 5-10. 